Recibido 05 de agosto, 2019 - Aceptado 05 de Sept, 2019

\title{
Influencia de la Disciplina Operativa durante el carguío en el rendimiento de la emulsión encartuchada 1 1/4 - acceso 575 Mina Ticlio 2018
}

\section{Influence of the Operative Discipline during the charge in the performance of the emulsion encartuchada 1 1/4" - access 575 Ticlio 2018}

\author{
Armando Picoy, Jose Sosa, Gladis Lazo ${ }^{3}$
}

\section{RESUMEN}

Para iniciar el estudio se realizó una revisión bibliográfica de los antecedentes relacionados a estudios de investigación sobre la influencia de la disciplina operativa durante el carguío y su rendimiento de las emulsiones encartuchadas, ante tal hecho históricamente se han realizado estudios experimentales sobre el comportamiento de los explosivos en base a nitrocarbonitratos (NCN) en la gama de emulsiones, estas mezclas explosivas presentan comportamientos variables en sus características técnicas principalmente en la velocidad de detonación influenciado por diferentes factores y condiciones de operación.

Para nuestro caso se ha evaluado el rendimiento de la emulsión encartuchada en diámetro de 1 1/4", trabajando en condiciones normales y rutinarias de operación; habiéndose evaluado en total 40 eventos - disparos en el Acceso 575 de la Mina Ticlio. Por cada evento se han tomado muestras de los procedimientos y conductas operacionales y al mismo tiempo se ha realizado la medición de la velocidad de detonación, utilizando un equipo hardware Microtrap de MREL y un sensor resistivo de $10 \mathrm{ohm} / \mathrm{m}$ probe-cable, obteniendo los resultados para cada evento.

Finalmente, el estudio consistió en demostrar las diferencias entre los resultados en la velocidad de detonación de la emulsión encartuchada de diámetro 1 1/4" supeditados a los escenarios de procedimientos y conductas operacionales denominado disciplina operativa.

Palabra Clave: Explosivo, emulsiones, disciplina operativa, velocidad de detonación.

1. Armando Picoy Almerco, Ingeniero de Minas Email: apicoy.in@gmail.com

2. José Luis Sosa Sánchez, Ingeniero de Sistemas. Mg Gestión del Sistema Ambiental. Email: jsosasa@undac.edu.pe

3. Gladis Lida Lazo Camposano, Ingeniero Electricista. Mg Administración con mención en Finanzas Email: g_lazo@uap.edu.pe 


\section{ABSTRACT}

To initiate the study, a bibliographic review of the background related to research studies on the influence of operational discipline during loading and its performance of hooded emulsions was performed, in the face of this fact historically experimental studies have been conducted on the behavior of explosives Based on nitrocarbonitrates $(\mathrm{NCN})$ in the range of emulsions, these explosive mixtures have variable behaviors in their technical characteristics, mainly in the detonation velocity influenced by different factors and operating conditions.

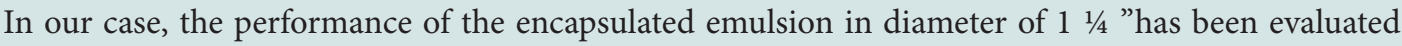
working under normal and routine operating conditions; In total, 40 events have been evaluated - shots in Access 575 of the Ticlio Mine, for each event samples of the procedures and operational behaviors have been taken and at the same time the detonation velocity measurement has been performed using a Microtrap hardware device MREL and a measurement sensor (Probe-cable with resistance of $10 \mathrm{ohm} / \mathrm{m}$ ), thus obtaining the results for each sampled event.

Finally, the study consisted in demonstrating the differences between the results in the detonation speed of the $1 \frac{1 / 4}{4}$ "diameter emulsion subject to the operational procedures and behavior scenarios called operational discipline.

Keyword: Explosive, emulsions, operational discipline, detonation speed.

\section{INTRODUCCIÓN}

Los fenómenos de detonación en los explosivos y el rendimiento de las emulsiones explosivas, vienen siendo estudiados ampliamente hasta la actualidad; es por ello que consideramos continuar con estos estudios, puesto que hoy en día las tecnologías en ingeniería de explosivos y voladuras se puede comprender mejor con el comportamiento de cada una de las propiedades físico-químicas de los explosivos.

Este documento conlleva la propuesta de demostrar que los operadores mineros en sus actividades rutinarias de carguío y voladura, utilizan las emulsiones encartuchadas de diámetro 1 1/4" donde se han observado prácticas, procedimientos y conductas operacionales durante cuatro (4) escenarios diferentes, realizados de manera aleatoria, en algunos casos supeditados por su conducta y en otros por desconocimiento de los operadores de carguío.
El presente estudio contempla el planteamiento metodológico que consiste en la definición del problema que se pretende abordar a través de la investigación; en este punto se delimita el objeto de estudio y se da a conocer las interrogantes o las grandes preguntas que orientan la investigación; también se contempla el marco teórico que consiste en la recopilación de antecedentes, investigaciones previas y consideraciones teóricas, con los cuales se sustenta el proyecto de investigación, análisis, hipótesis o experimento, permitiendo la interpretación de sus resultados y la formulación de conclusiones.

Por último se incluye con el contraste de la hipótesis, procedimiento que nos permite juzgar si la muestra representa a la población que ala vez origina la discusión de resultados donde se explica el comportamiento de sus variables para establecer las conclusiones y recomendaciones finales. 


\section{MÉTODOS Y MATERIALES}

El método empleado en la presente investigación es el científico, por lo que antes debe ocurrir el proceso de la voladura para proceder con la observación de la velocidad de detonación a través de un equipo para adquisición de datos Microtrap de MREL conllevando a la formulación del problema, hipótesis, verificación, análisis y conclusión como consta en el trabajo (Fideas, 2006).

\section{Instrumentos para recolección de datos}

Instrumento utilizado para la variable 1 - disciplina operativa durante el carguío.

El instrumento empleado basado en un formulario de observación planeada de tareas (OPT) para controles operacionales detallado en la figura 1; instrumento confeccionado en concordancia con la NTP 386 "Observaciones planeadas del trabajo - Instituto Nacional de Seguridad e Higiene en el Trabajo - Ministerio de Trabajo y Asuntos Sociales España”, donde el verificador solo puede marcar uno de los escenarios con un aspa (X); en caso de marcar más de una alternativa, se invalida el ítem.

\begin{tabular}{|c|c|c|c|c|}
\hline & \multicolumn{3}{|c|}{ OBSERVACION PLANEADA DE TAREAS } & VERSION \\
\hline & \multicolumn{3}{|c|}{ CONT ROLES OPERA CIONALES } & 1.0 \\
\hline Unida d: & Área: & Nivel: & Fecha: & \\
\hline Lugar: & & Zona: & $\mathrm{N}^{\circ}$ asistentes & \\
\hline
\end{tabular}

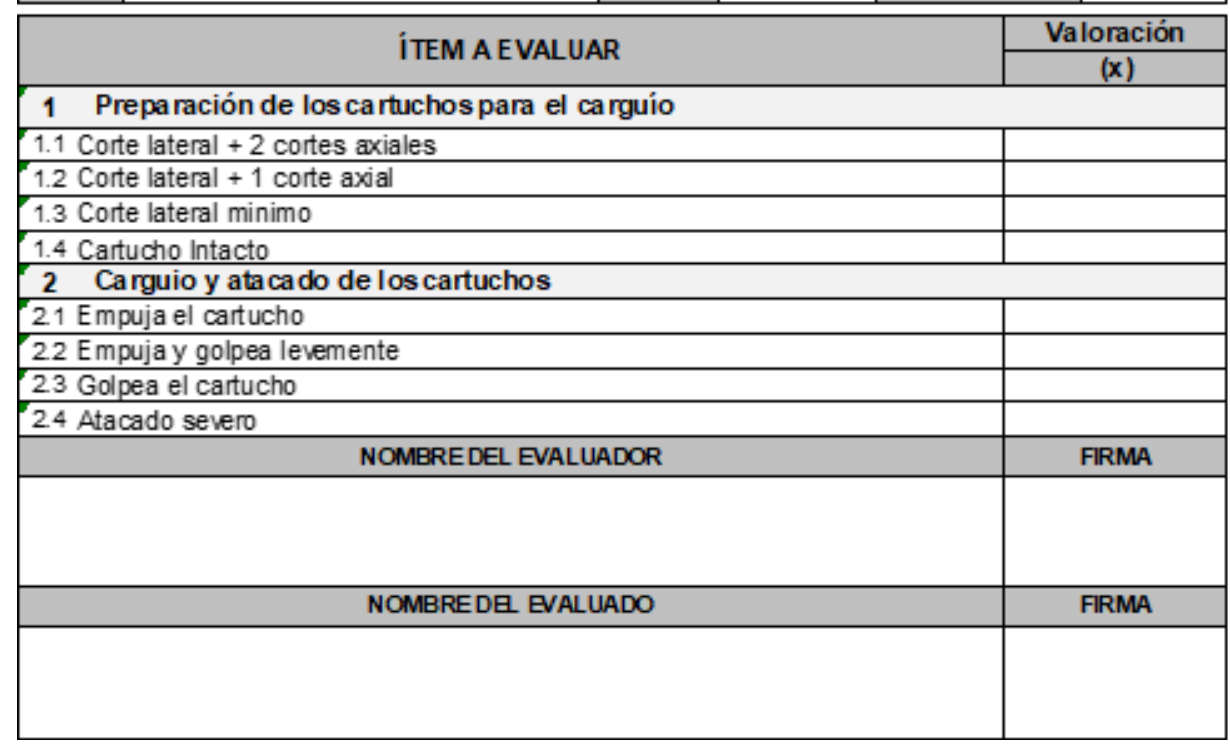

Figura $\mathrm{N}^{\circ} 1$. Instrumento de recolección de datos para la disciplina operativa

Fuente: Elaboración propia

\section{Objetivo}

Obtener información acerca de la disciplina operativa durante el carguío en el Acceso 575 de la Mina Ticlio.

\section{Estructura}

Las dimensiones que evalúa la disciplina operativa se detallan en la Tabla 1 y 2. 
Tabla $\mathrm{N}^{\circ} 1$. Especificaciones para verificación de la Disciplina Operativa.

\begin{tabular}{|c|c|c|c|}
\hline Dimensiones & $\begin{array}{c}\text { Estructura } \\
\text { Items }\end{array}$ & Total & Porcentaje \\
\hline Preparación de los & \multirow{2}{*}{$1,1-1,2-1,3-1,4$} & \multirow{2}{*}{4} & \multirow{2}{*}{$50 \%$} \\
\hline $\begin{array}{l}\text { Cartuchos } \\
\text { Acoplamiento de los }\end{array}$ & & & \\
\hline $\begin{array}{l}\text { Acoplamiento de los } \\
\text { explosivos en el taladro }\end{array}$ & $2,1-2,2-2,3-2,4$ & 4 & $50 \%$ \\
\hline \multicolumn{2}{|c|}{ TOTAL } & 8 & $100 \%$ \\
\hline
\end{tabular}

Tabla N². Baremos de la lista de verificación de la Disciplina Operativa.

\begin{tabular}{cccccc}
\hline \multicolumn{2}{c}{\begin{tabular}{c} 
Preparación de la \\
\multicolumn{2}{c}{ carga explosiva }
\end{tabular}} & \multicolumn{2}{c}{$\begin{array}{c}\text { Acoplamiento de los } \\
\text { explosivos en el }\end{array}$} & \multicolumn{2}{c}{$\begin{array}{c}\text { Disciplina Operativa } \\
\text { durante el carguio }\end{array}$} \\
\hline Muy Bueno & $6-7$ & Optimo & $7-8$ & Muy Satisfactorio & $12-15$ \\
Regular & $3-5$ & Aceptable & $3-6$ & Satisfactorio & $5-11$ \\
Deficiente & $0-2$ & Deficiente & $0-2$ & Insatisfactorio & $1-4$ \\
\hline
\end{tabular}

Instrumento para la variable 2 - rendimiento de la emulsión encartuchada de 1 1/4”

El instrumento empleado está basado en un indicador de rendimiento y eficiencia del explosivo en el estado de detonación a través de la velocidad de detonación (VOD) el que se observa en la Figura 2, parámetro utilizado para dimensionar e inferir otros parámetros como la presión de detonación, presión de taladro, la capacidad de trabajo (potencia mecánica); con alcance al modelo predictivo de fragmentación (Kuz-Ram) que, para nuestra investigación es un indicador de calidad en el rendimiento del explosivo lo que justifica su clasificación:

- Detonantes de bajo régimen cuando su velocidad de detonación esta entre $1000 \mathrm{~m} / \mathrm{s}$ a $2000 \mathrm{~m} / \mathrm{s}$ (transición entre deflagración y detonación).

- Detonantes de régimen normal cuando su velocidad de detonación esta entre $2000 \mathrm{~m} / \mathrm{s}$ a $5000 \mathrm{~m} / \mathrm{s}$.

- Detonantes de alto régimen cuando su velocidad de detonación está por encima de $5000 \mathrm{~m} / \mathrm{s}$.

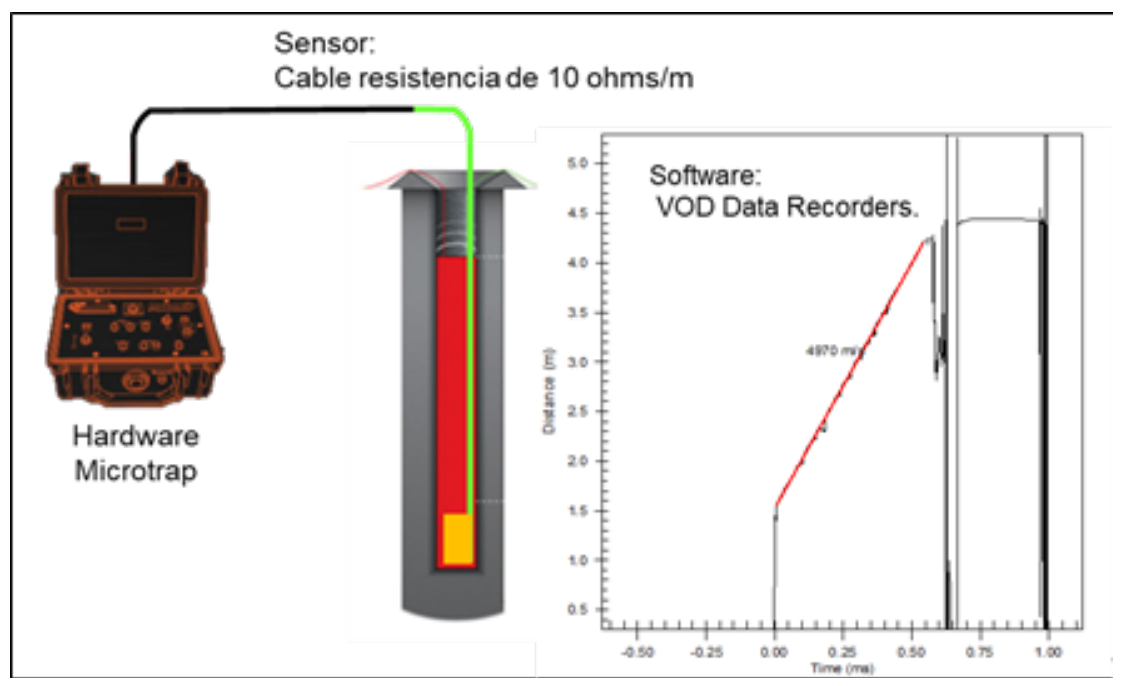

Figura $\mathrm{N}^{\circ} 2$. Indicador de rendimiento del explosivo 


\section{Objetivo}

Obtener información acerca del rendimiento de las emulsiones encartuchadas 1 1/4" mediante la velocidad de detonación.

\section{Estructura}

La dimensión que evalúa el rendimiento de la emulsión encartuchada se detalla en la Tabla 3.

Tabla No3. Niveles de rendimiento de las emulsiones encartuchadas en 1 1/4".

\begin{tabular}{|lccc}
\hline Niveles & REGIMEN ALTO & $\begin{array}{c}\text { REGIMEN NORMAL } \\
\text { SUPERIOR }\end{array}$ & $\begin{array}{c}\text { REGIMEN NORMAL } \\
\text { BAIO }\end{array}$ \\
\hline $\begin{array}{l}\text { Rendimiento de la Emulsion } \\
\text { Encartuchada 11/4" }\end{array}$ & $5200-5001$ & $5000-4801$ & $4800-4600$ \\
\hline
\end{tabular}

\section{RESULTADOS}

Proceso de contraste de hipótesis

En el presente rubro se pone de manifiesto la relación existente entre las variables en estudio, contrastándolas en el mismo orden que han sido formuladas, con el fin de facilitar la interpretación de los datos, ver Tabla 4.

Tabla N4. Parámetros para el contraste de hipótesis

\begin{tabular}{ll}
\hline & CONTRASTE DE HIPOTESIS \\
\hline Ho: & No existe influencia significativa \\
H1: & Existe influencia significativa \\
Nivel de significancia: & $\mathrm{a}=0.05$ \\
Valor estadístico de & Coeficiente de Correlación Chi \\
prueba: & Cuadrado. \\
\hline
\end{tabular}

\section{Prueba de hipótesis general}

Existe influencia significativa de la disciplina operativa durante el carguío en el rendimiento de la emulsión encartuchada 1 1⁄4", acceso 575 Ticlio 2018.

\section{Tabla N ${ }^{\circ}$. Contingencia Disciplina Operativa por Rendimiento emulsiones encartuchadas} de 1 1/4"

\begin{tabular}{|c|c|c|c|c|c|c|c|c|c|}
\hline \multirow{4}{*}{$\begin{array}{l}\text { Disciplina } \\
\text { Operativa }\end{array}$} & \multicolumn{7}{|c|}{ Rendimiento de la Emulsion Encartuchada 1 1/4" } & & \multirow{4}{*}{ Total } \\
\hline & \multicolumn{4}{|c|}{ REGIMEN NORMAL } & \multicolumn{3}{|c|}{ REGIMEN ALTO } & & \\
\hline & \multicolumn{2}{|c|}{$\begin{array}{c}\text { BAJO } \\
(4600-4800)\end{array}$} & \multicolumn{2}{|c|}{$\begin{array}{c}\text { SUPERIOR } \\
(4801-5000)\end{array}$} & \multicolumn{2}{|c|}{ (5001 - 5200) } & \multirow[t]{2}{*}{$(\mathrm{m} / \mathrm{s})$} & & \\
\hline & $\mathrm{n}$ & $\%$ & $\mathrm{n}$ & $\%$ & $\mathrm{n}$ & $\%$ & & & \\
\hline & 4 & & 4 & & 4 & & & & \\
\hline Insatisfactorio & $\rightarrow$ & $22.50 \%$ & t & & & & & 9 & $22.5 \%$ \\
\hline Satisfactorio & & $\longrightarrow$ & 24 & $60 \%$ & | & & & 24 & $60.0 \%$ \\
\hline Muy Satisfactorio & & & & & 7 & $18 \%$ & & 7 & $17.5 \%$ \\
\hline Total & 9 & $23 \%$ & 24 & $60 \%$ & 7 & $18 \%$ & & 40 & $100.0 \%$ \\
\hline
\end{tabular}


Interpretando la Tabla 5 de contingencia se observó lo siguiente:

- El 60\% de los operadores de carguío, muestran un nivel de disciplina operativa "satisfactorio", lo cual tiene relación con el rendimiento de la emulsión encartuchada $11 / 4$ ", por alcanzar velocidades de detonación en el rango de 4800 a 5000 $\mathrm{m} / \mathrm{s}$, ubicándolos en "régimen normal superior".

- El 22.5\% de los operadores de carguío, muestran un nivel de disciplina operativa "insatisfactorio", lo cual tiene relación con el rendimiento de la emulsión encartuchada $1 \frac{1 / 4}{4}$, por alcanzar velocidades de detonación en el rango de 4600 a $4800 \mathrm{~m} / \mathrm{s}$, ubicándolos en "régimen normal superior".
- El 17.5\% de los operadores de carguío, muestran un nivel de disciplina operativa "muy satisfactorio", lo cual tiene relación con el rendimiento de la emulsión encartuchada $1 \frac{1 / 4}{4}$, por alcanzar velocidades de detonación mayor a los $5000 \mathrm{~m} / \mathrm{s}$, ubicándolos en "régimen alto".

Asumiendo un valor $\mathrm{p}=0,05$; se rechaza la hipótesis nula y se acepta la hipótesis alterna señalando que "Existe influencia significativa de la disciplina operativa durante el carguío en el rendimiento de la emulsión encartuchada $1 \frac{1 / 4}{}$ ” en el acceso 575 Ticlio 2018 "

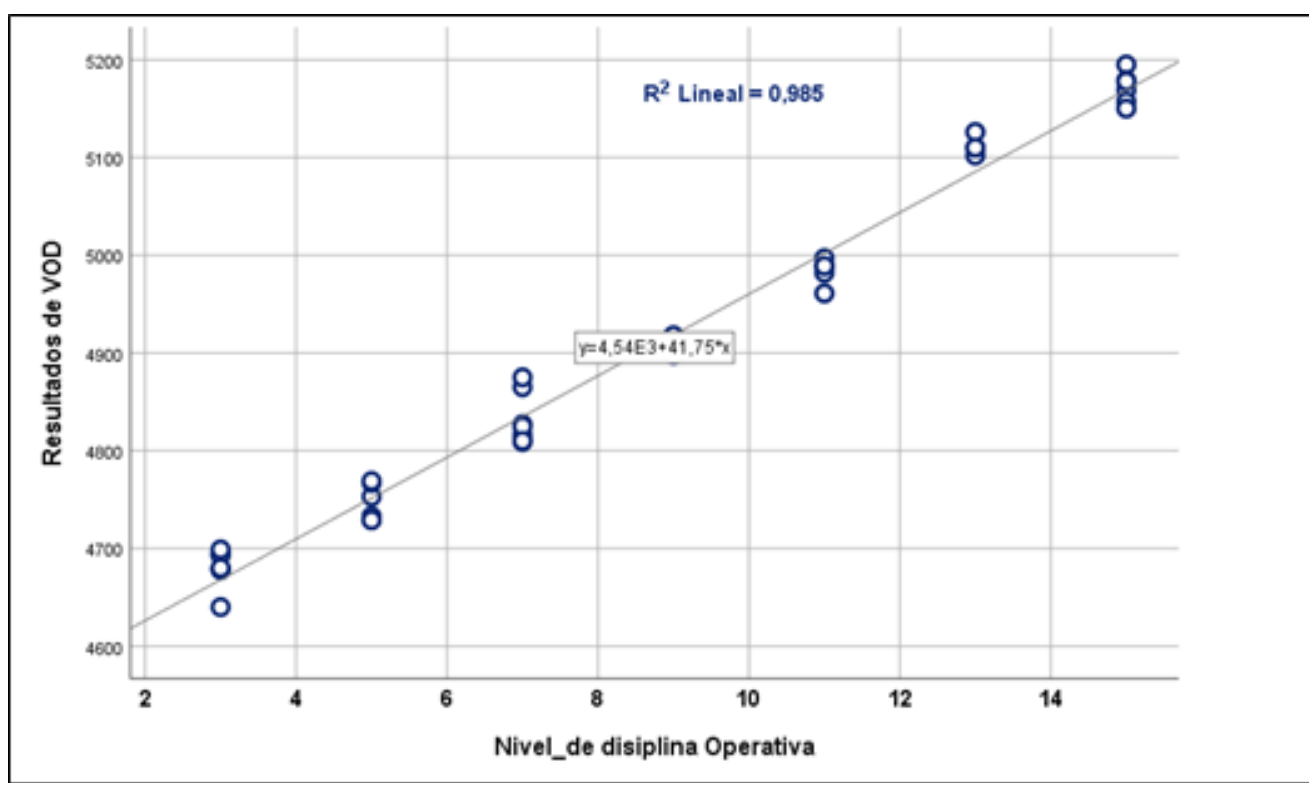

Gráfico $\mathrm{N}^{\circ} 1$. Correlación entre la disciplina operativa y el rendimiento de la emulsión encartuchada $1 \frac{1 / 4}{}$ "

Acorde al Gráfico 1, la disciplina operativa durante el carguío presenta una alta correlación $(\mathrm{R} 2=$ 0.985 ) con el rendimiento de la emulsión encartuchada $1 \frac{114}{4}$, es decir que a mayores niveles de la disciplina operativa existirán mayores niveles de rendimiento de las emulsiones encartuchadas en 1 1/4". Prueba de hipótesis específica

\section{Hipótesis específica 1}

Existe influencia significativa de la preparación de cartuchos durante el carguío en el rendimiento de la emulsión encartuchada de 11/4", acceso 575 - Ticlio 2018. 
Tabla $\mathrm{N}^{\circ} 6$. Contingencia dimensión preparación de la carga explosiva por el rendimiento de las emulsiones encartuchadas de 1 1/4"

\begin{tabular}{|c|c|c|c|c|c|c|c|c|c|}
\hline \multirow{5}{*}{$\begin{array}{l}\text { Dimensión } \\
\text { Preparación de } \\
\text { la carga explosiva }\end{array}$} & \multicolumn{7}{|c|}{ Rendimiento de la Emulsion Encartuchada 11/4" } & & \multirow{5}{*}{ Total } \\
\hline & \multicolumn{4}{|c|}{ REGIMEN NORMAL } & \multicolumn{3}{|c|}{ REGIMEN ALTO } & & \\
\hline & \multirow{2}{*}{\multicolumn{2}{|c|}{$\begin{array}{c}\text { BAJO } \\
(4600-4800)\end{array}$}} & \multirow{2}{*}{\multicolumn{2}{|c|}{$\begin{array}{c}\text { SUPERIOR } \\
(4801 \cdot 5000)\end{array}$}} & \multirow{2}{*}{\multicolumn{2}{|c|}{$(5001-5200)$}} & \multirow{3}{*}{$(\mathrm{m} / \mathrm{s})$} & & \\
\hline & & & & & & & & & \\
\hline & n & $\%$ & $\mathrm{n}$ & $\%$ & $\mathrm{n}$ & $\%$ & & & \\
\hline & $\uparrow$ & & 4 & & $\uparrow$ & & & & \\
\hline Deficlente & $\rightarrow$ & $20.00 \%$ & | & & & & & 8 & $20.0 \%$ \\
\hline Regular & & $\longrightarrow$ & 21 & $53 \%$ & 1 & & & 21 & $52.5 \%$ \\
\hline Muy Bueno & & & & & $\rightarrow 11$ & $28 \%$ & & 11 & $27.5 \%$ \\
\hline Total & 8 & $20 \%$ & 21 & $53 \%$ & 11 & $28 \%$ & & 40 & $100.0 \%$ \\
\hline
\end{tabular}

Interpretando la Tabla 6 de contingencia se observó lo siguiente:

- El 52.5\% de los operadores de carguío muestran un nivel de preparación de la carga explosiva "regular"; elemento que tiene relación con el rendimiento de la emulsión encartuchada $1 \frac{1 / 4}{\text { ", }}$ ubicándolos en "régimen normal superior" por alcanzar velocidades de detonación en el rango de 4801 a $5000 \mathrm{~m} / \mathrm{s}$.

- El 27.5\% de los operadores de carguío muestran un nivel de preparación de la carga explosiva "muy bueno"; elemento que tiene relación con el rendimiento de la emulsión encartuchada 1 1/4", ubicándolos en "régimen alto" por alcanzar velocidades de detonación mayores a $5000 \mathrm{~m} / \mathrm{s}$.

- El 20\% de los operadores de carguío muestran un nivel de preparación de la carga explosiva "deficiente"; situándolos en "régimen normal bajo" por alcanzar velocidades de detonación en el rango de 4600 a $4800 \mathrm{~m} / \mathrm{s}$.

Asumiendo el valor $\mathrm{p}=0,050$, se rechaza la hipótesis nula y se acepta la hipótesis de alterna; entonces la preparación de la carga explosiva se relaciona significativamente con el rendimiento de las emulsiones encartuchadas $1 \frac{1 / 4}{4}$ en el acceso 575 Ticlio 2018.

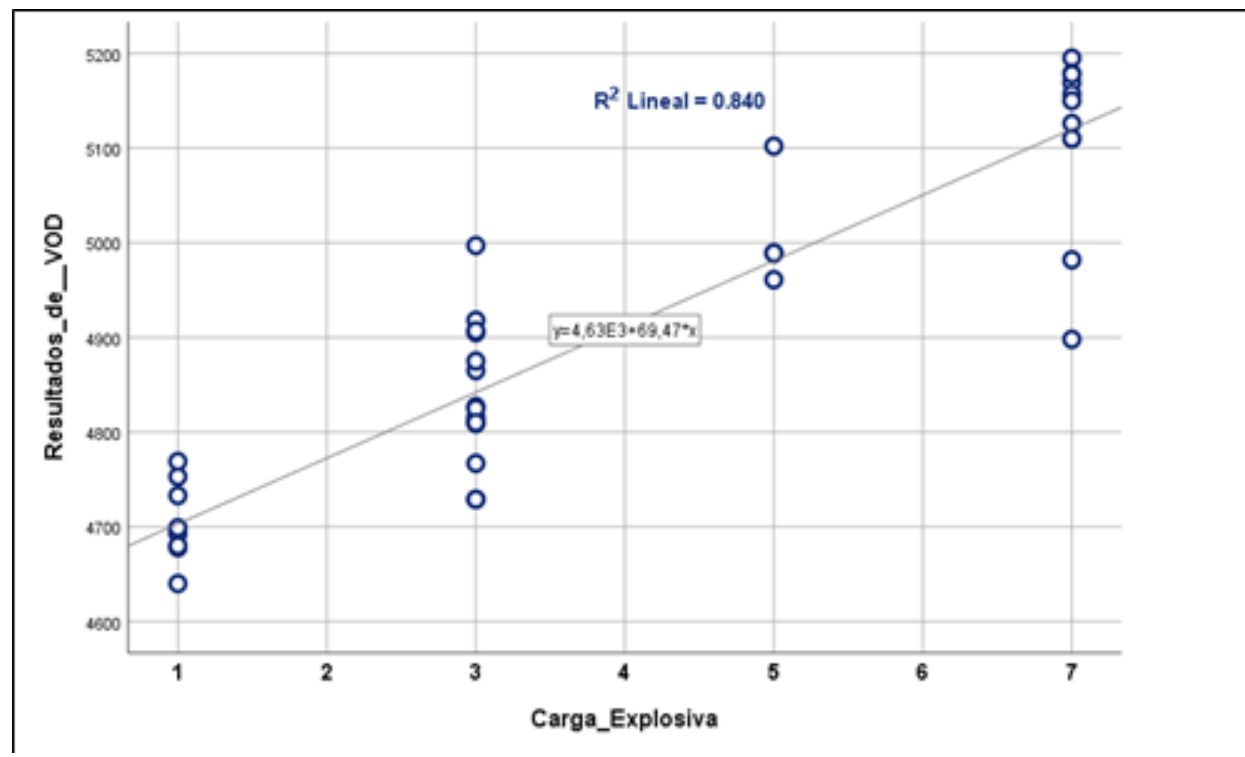

Gráfico $\mathrm{N}^{\circ}$ 2. Correlación entre la preparación de la carga explosiva y el rendimiento de la emulsión encartuchada 1 1/4" 
Acorde al Gráfico 3, el acoplamiento de los explosivos en el taladro presenta una buena correlación $(\mathrm{R} 2=0.832)$ con el rendimiento de la emulsión encartuchada $1 \frac{1 / 4}{4}$, es decir que a mayores niveles de acoplamiento de los explosivos en el taladro existirán mayores niveles de rendimiento de las emulsiones encartuchadas $1 \frac{1 / 4}{}$ ".

\section{DISCUSIÓN}

Luego del análisis de los resultados se pudo hallar que la disciplina operativa se relaciona significativamente con el rendimiento de las emulsiones encartuchadas de 1 1/4" en el acceso 575 Mina Ticlio - 2018; por lo que se define a la disciplina operativa como el cumplimiento riguroso y continuo de todos los procedimientos e instrucciones de trabajo, tanto operativos, administrativos y de mantenimiento de un centro de trabajo, a través del proceso de tenerlos disponibles con la mejor calidad, cumplimiento y comunicarlos de forma efectiva a quienes lo aplican y de exigir su cumplimiento estricto (Guía de disciplina operativa PEMEX - Petróleos Mexicanos - 1983), durante nuestro estudio de observación respecto a la preparación de la carga explosiva y el acoplamiento de los explosivos; también se ha podido establecer que se relacionan significativamente con la citada variable, puesto que se presenta hasta cuatro escenarios posibles, supeditados a las prácticas y conductas operacionales teniendo como resultado una variación cualitativa en dichas dimensiones y sus efectos en el rendimiento de las emulsiones encartuchadas 11/4", conclusiones que concuerdan con lo especificado por Calderon, M. (2015); quien realizó un estudio sobre la optimización de las prácticas de perforación y voladura donde una de sus conclusiones indica que el trabajo que se realiza en las operaciones unitarias donde están implicados desde un obrero hasta el superintendente es muy importante, ya que todos tienen el mismo fin de realizar la perforación y voladura de manera correcta y sin incidentes en avance y producción; es decir las buenas practicas tiende a niveles aceptables en la perforación y voladura; en relación con nuestro caso la voladura es un término genérico utilizado para referirse a la actividad de carguío y disparo (procedimientos antes, durante) en tal sentido se deduce que se han presentado dificultades en las dimensiones de preparación de la carga explosiva y acoplamiento de los explosivos.

\section{CONCLUSIONES}

Con un nivel de confianza del 95\% y un margen de error del $5 \%$ se concluye que:

a) Se concluye que la influencia de la disciplina operativa durante el carguío en el rendimiento de la emulsión encartuchada de 1/1/4" en el acceso 575 Ticlio 2018 ha sido positiva; sin embargo debemos mencionar que sólo el $22.5 \%$ han alcanzado el nivel máximo deseado "muy satisfactorio"; en tanto que el $60 \%$ de las pruebas alcanzó el nivel de progreso "satisfactorio"; por lo tanto a medida que mejora el nivel de disciplina operativa durante el carguío, mejora el rendimiento de la emulsión encartuchadas en 11/4".

b) Se identificó que la influencia de la preparación de carga explosiva en el rendimiento de la emulsión encartuchada de 11/4" fue positiva, determinándose que sólo un $28 \%$ de las pruebas ha alcanzado un nivel de progreso de "muy bueno" y un 53\% un nivel de progreso "regular"; por lo que podemos afirmar que en función al diseño de la emulsión encartuchada, es necesario alcanzar el nivel muy bueno facilitando el acoplamiento de explosivo y la trasmisión de onda de detonación contribuyendo a incrementar el rendimiento de la emulsión encartuchadas en 11/4".

c) Se ha demostrado la influencia del acoplamiento de los explosivos en el taladro en el rendimiento de la emulsión encartuchada 11/4"; que igualmente ha sido positiva; puesto que un $20 \%$ ha alcanzado el nivel "optimo" y el 55\% el nivel de "aceptable"; debido a sus componentes de la masa explosiva, el componente sensibilizador es vital para inducir la detonación-explosión; siendo necesario alcanzar el nivel óptimo minimizando la destrucción y reducción del elemento sensibilizador durante el atacado. contribuyendo 
a incrementar el rendimiento de la emulsión encartuchadas en $1 \frac{11}{4} "$

\section{BIBLIOGRAFÍA}

Caisaguano, J., \& Armando, M. (2000). Caracterizacion del poder de iniciación de multiplicadores . Ecuador: Escuela Superior Politecnica del Litoral .

Fideas, A. (2006). Proyecto de la investigación cientifífica. Caracas: Episteme.

Johansson, L. (2011). Numerical Study of Non Ideal Explosive Detonatión. USA: Luleá University of Tecnology. Linares Carrasco, N. S. (2013). Estudio sobre la medida de la velocidad de detonacion. Madrid: Universidad Politecnica de Madrid.

Linares Carrasco, N. S. (2013). Estudio sobre la medida de la velocidad de detonación. España: Universidad Politecnica de Madrid.

Linares Carrasco, N. S. (2013). Estudio sobre la medida de la velocidad de detonación. España: Universidad Politecnica de Madrid.

Medina Cortez, R. O. (2014). Evaluación tecnico económico ecológico de los resultados de las pruebas realizadas usando emulsiones gasificadas en Cuajone. Perú: Universidad Nacional de Ingenieria .

MREL GROUP OF COMPANIES LIMITED. (01 de FEBRERO de 2013). mrel.com/blasting.../files/manuals/ MicroTrap_Operations_Manual_Spanish.pdf. Obtenido de mrel.com/blasting.../files/manuals/MicroTrap_ Operations_Manual_Spanish.pdf.

Rodriguez Polo, L. S. (2009). Sistema de medición de velocidad y tiempo de detonación para explosivos usando el método de fibras ópticas. Perú: Pontificia Universidad Catolica.

Scherpenisse, C. (2006). Formula de calculo de la presion de taladro. Curso Internacional de análisis y modelamiento de vibraciones para el control de daño al macizo rocoso. . Chile: ASP Blastronics. 


\section{ANEXOS}

Tabla 8: Datos para la prueba de hipótesis

\begin{tabular}{|c|c|c|c|c|}
\hline $\begin{array}{c}\text { Número de } \\
\text { observaciones }\end{array}$ & $\begin{array}{l}\text { Nivel de } \\
\text { Disciplina } \\
\text { Operativa }\end{array}$ & $\begin{array}{c}\text { Preparación } \\
\text { carga } \\
\text { explosiva }\end{array}$ & $\begin{array}{c}\text { Acoplamiento } \\
\text { de } \\
\text { explosivos }\end{array}$ & $\begin{array}{l}\text { Resultados } \\
\text { de VOD }\end{array}$ \\
\hline 1 & 15 & 7 & 8 & 5169 \\
\hline 2 & 3 & 1 & 2 & 4694 \\
\hline 3 & 11 & 5 & 6 & 4989 \\
\hline 4 & 9 & 3 & 6 & 4918 \\
\hline 5 & 7 & 3 & 4 & 4809 \\
\hline 6 & 15 & 7 & 8 & 5157 \\
\hline 7 & 7 & 3 & 4 & 4827 \\
\hline 8 & 13 & 5 & 8 & 5102 \\
\hline 9 & 11 & 5 & 6 & 4961 \\
\hline 10 & 7 & 3 & 4 & 4865 \\
\hline 11 & 15 & 7 & 8 & 5195 \\
\hline 12 & 3 & 1 & 2 & 4679 \\
\hline 13 & 13 & 7 & 6 & 5110 \\
\hline 14 & 3 & 1 & 2 & 4678 \\
\hline 15 & 7 & 3 & 4 & 4816 \\
\hline 16 & 15 & 7 & 8 & 5150 \\
\hline 17 & 9 & 3 & 6 & 4905 \\
\hline 18 & 11 & 7 & 4 & 4982 \\
\hline 19 & 5 & 1 & 4 & 4753 \\
\hline 20 & 3 & 1 & 2 & 4693 \\
\hline 21 & 15 & 7 & 8 & 5179 \\
\hline 22 & 5 & 3 & 2 & 4729 \\
\hline 23 & 11 & 3 & 8 & 4997 \\
\hline 24 & 13 & 7 & 6 & 5126 \\
\hline 25 & 5 & 3 & 2 & 4767 \\
\hline 26 & 5 & 1 & 4 & 4733 \\
\hline 27 & 15 & 7 & 8 & 5178 \\
\hline 28 & 5 & 3 & 2 & 4729 \\
\hline 29 & 5 & 1 & 4 & 4769 \\
\hline 30 & 7 & 3 & 4 & 4812 \\
\hline 31 & 13 & 7 & 6 & 5110 \\
\hline 32 & 11 & 5 & 6 & 4989 \\
\hline 33 & 7 & 3 & 4 & 4875 \\
\hline 34 & 3 & 1 & 2 & 4640 \\
\hline 35 & 7 & 3 & 4 & 4825 \\
\hline 36 & 3 & 1 & 2 & 4680 \\
\hline 37 & 7 & 3 & 4 & 4810 \\
\hline 38 & 9 & 7 & 2 & 4898 \\
\hline 39 & 3 & 1 & 2 & 4699 \\
\hline 40 & 9 & 3 & 6 & 4907 \\
\hline
\end{tabular}

Fuente: Instrumentos de medición. 
Tabla 9: Análisis de datos del instrumento Disciplina Operativa - Preparación de la carga

\begin{tabular}{|c|c|c|c|c|c|c|c|c|c|}
\hline 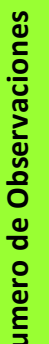 & 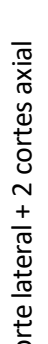 & 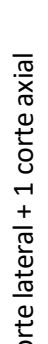 & 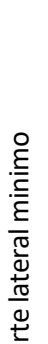 & 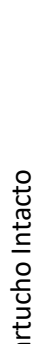 & 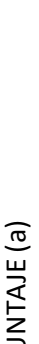 & 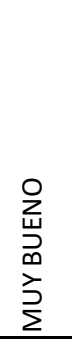 & 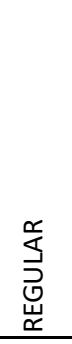 & $\begin{array}{l}\frac{w}{\Sigma} \\
\frac{w}{\frac{u}{u}} \\
\text { 岁 }\end{array}$ & \\
\hline $\mathbf{z}$ & & త & & & 근 & 7 a 6 & 5 a 3 & 2 a 1 & NIVEL \\
\hline 1 & $x$ & & & & 7 & 1 & & & MUY BUENO \\
\hline 2 & & & & $x$ & 1 & & & 1 & DEFICIENTE \\
\hline 3 & & $\mathrm{x}$ & & & 5 & & 1 & & REGULAR \\
\hline 4 & & & $\mathrm{x}$ & & 3 & & 1 & & REGULAR \\
\hline 5 & & & $x$ & & 3 & & 1 & & REGULAR \\
\hline 6 & $x$ & & & & 7 & 1 & & & MUY BUENO \\
\hline 7 & & & $x$ & & 3 & & 1 & & REGULAR \\
\hline 8 & & $\mathrm{x}$ & & & 5 & & 1 & & REGULAR \\
\hline 9 & & $x$ & & & 5 & & 1 & & REGULAR \\
\hline 10 & & & $x$ & & 3 & & 1 & & REGULAR \\
\hline 11 & $x$ & & & & 7 & 1 & & & MUY BUENO \\
\hline 12 & & & & $x$ & 1 & & & 1 & DEFICIENTE \\
\hline 13 & $x$ & & & & 7 & 1 & & & MUY BUENO \\
\hline 14 & & & & $x$ & 1 & & . & 1 & DEFICIENTE \\
\hline 15 & & & $\mathrm{x}$ & & 3 & & 1 & & REGULAR \\
\hline 16 & $x$ & & & & 7 & 1 & & & MUY BUENO \\
\hline 17 & & & $\mathrm{x}$ & & 3 & & 1 & & REGULAR \\
\hline 18 & $\mathrm{x}$ & & & & 7 & 1 & & & MUY BUENO \\
\hline 19 & & & & $x$ & 1 & & & 1 & DEFICIENTE \\
\hline 20 & & & & $x$ & 1 & & & 1 & DEFICIENTE \\
\hline 21 & $x$ & & & & 7 & 1 & 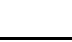 & & MUY BUENO \\
\hline 22 & & & $\mathrm{x}$ & & 3 & & 1 & & REGULAR \\
\hline 23 & & & $\mathrm{x}$ & & 3 & & 1 & & REGULAR \\
\hline 24 & $\mathrm{x}$ & & & & 7 & 1 & & & MUY BUENO \\
\hline 25 & & & $x$ & & 3 & & 1 & & REGULAR \\
\hline 26 & & & & $x$ & 1 & & & 1 & DEFICIENTE \\
\hline 27 & $x$ & & & & 7 & 1 & & & MUY BUENO \\
\hline 28 & & & $x$ & & 3 & & 1 & & REGULAR \\
\hline 29 & & & & $x$ & 1 & & & 1 & DEFICIENTE \\
\hline 30 & & & $x$ & & 3 & & 1 & & REGULAR \\
\hline 31 & $x$ & & & & 7 & 1 & & & MUY BUENO \\
\hline 32 & & $\mathrm{x}$ & & & 5 & & 1 & & REGULAR \\
\hline 33 & & & $x$ & & 3 & & 1 & & REGULAR \\
\hline 34 & & & & $x$ & 1 & & & 1 & DEFICIENTE \\
\hline 35 & & & $\mathrm{x}$ & & 3 & & 1 & & REGULAR \\
\hline 36 & & & & $x$ & 1 & & 1 & & REGULAR \\
\hline 37 & & & $x$ & & 3 & & 1 & & REGULAR \\
\hline 38 & $\mathrm{x}$ & & & & 7 & 1 & & & MUY BUENO \\
\hline 39 & & & & $x$ & 1 & & 1 & & REGULAR \\
\hline 40 & & & $\mathrm{x}$ & & 3 & & 1 & & REGULAR \\
\hline
\end{tabular}

Fuente: Instrumentos de medición. 
Tabla 10: Análisis de datos del instrumento Disciplina Operativa - Acoplamiento de los explosivos

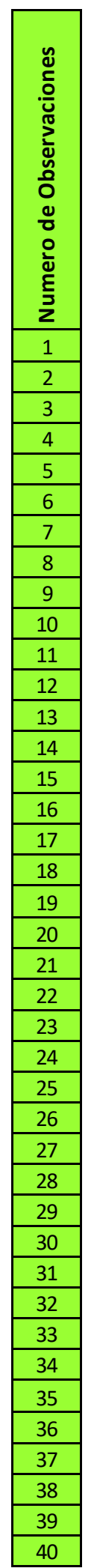

\begin{tabular}{|c|c|c|c|c|}
\hline 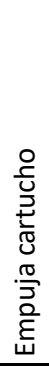 & 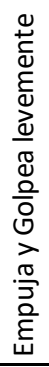 & 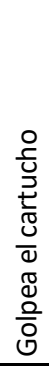 & 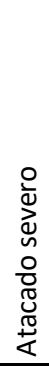 & 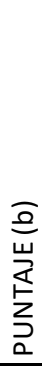 \\
\hline \multirow[t]{5}{*}{$\mathrm{x}$} & & & & 8 \\
\hline & & & $\mathrm{x}$ & 2 \\
\hline & $x$ & & & 6 \\
\hline & $x$ & & & 6 \\
\hline & & $\mathrm{x}$ & & 4 \\
\hline \multirow[t]{2}{*}{$\mathrm{x}$} & & & & 8 \\
\hline & & $x$ & & 4 \\
\hline \multirow[t]{3}{*}{$\mathrm{x}$} & & & & 8 \\
\hline & $x$ & & & 6 \\
\hline & & $x$ & & 4 \\
\hline \multirow[t]{5}{*}{$\mathrm{x}$} & & & & 8 \\
\hline & & & $x$ & 2 \\
\hline & $x$ & & & 6 \\
\hline & & & $x$ & 2 \\
\hline & & $x$ & & 4 \\
\hline \multirow[t]{5}{*}{$\mathrm{x}$} & & & & 8 \\
\hline & $x$ & & & 6 \\
\hline & & $x$ & & 4 \\
\hline & & $x$ & & 4 \\
\hline & & & $x$ & 2 \\
\hline \multirow[t]{2}{*}{$\mathrm{x}$} & & & & 8 \\
\hline & & & $x$ & 2 \\
\hline \multirow[t]{4}{*}{$x$} & & & & 8 \\
\hline & $x$ & & & 6 \\
\hline & & & $x$ & 2 \\
\hline & & $x$ & & 4 \\
\hline \multirow[t]{14}{*}{$x$} & & & & 8 \\
\hline & & & $\mathrm{x}$ & 2 \\
\hline & & $x$ & & 4 \\
\hline & & $x$ & & 4 \\
\hline & $x$ & & & 6 \\
\hline & $x$ & & & 6 \\
\hline & & $x$ & & 4 \\
\hline & & & $x$ & 2 \\
\hline & & $x$ & & 4 \\
\hline & & & $x$ & 2 \\
\hline & & $x$ & & 4 \\
\hline & & & $x$ & 2 \\
\hline & & & $x$ & 2 \\
\hline & $x$ & & & 6 \\
\hline
\end{tabular}

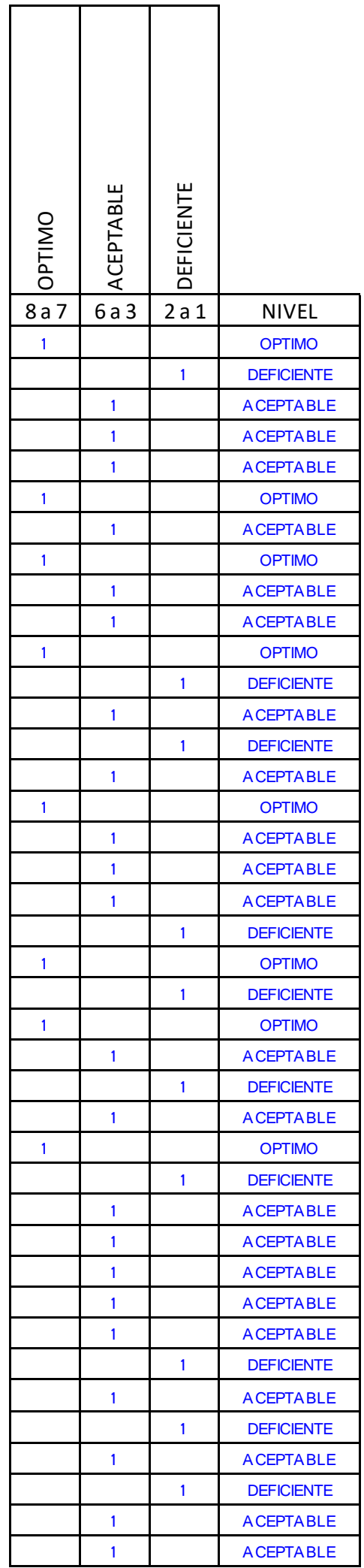

Fuente: Instrumentos de medición. 